\title{
Asymptotics of the Finite-time Ruin Probability for the Sparre Andersen Risk Model Perturbed by an Inflated Stationary Chi-process
}

\author{
Enkelejd Hashorva and Lanpeng Ji *
}

\begin{abstract}
In this paper we consider the Sparre Andersen risk model that is perturbed by an inflated chiprocess with non-negative random inflator $R$. Under some conditions on the perturbation and the random inflator, which allow for both small and large fluctuations, exact asymptotic behaviour of the finite-time ruin probability is obtained when initial reserve tends to infinity.
\end{abstract}

Key words: Sparre Andersen risk model; chi-process; Gaussian process; perturbed risk process; finite-time ruin probability; subexponential distribution.

\section{Introduction}

Consider a claim counting process $N(t)=\sum_{i=1}^{\infty} \mathbb{I}\left(\tau_{1}+\cdots+\tau_{i} \leq t\right)$, where $\tau_{i}, i \in \mathbb{N}$, are the i.i.d. claim inter-arrival times with distribution function $F_{\tau}$ and mean $\mathbb{E}\left(\tau_{1}\right)=1 / \lambda, \lambda>0$; here $\mathbb{I}(\cdot)$ denotes the indicator function. Let $X_{i}, i \in \mathbb{N}$, be i.i.d. non-negative random variables, representing the claim sizes. In risk theory, for a given portfolio of risks, the insurer's claim surplus process is defined by

$$
S(t)=\sum_{i=1}^{N(t)} X_{i}-c t, \quad t \geq 0,
$$

where $\sum_{i=1}^{N(t)} X_{i}$ is the aggregate claim process and $c(>0)$ is the premium rate assumed to be a constant. Suppose further that $\{N(t), t \geq 0\}$ and $X_{i}, i \in \mathbb{N}$, are independent, and $c>\lambda \mathbb{E}\left(X_{1}\right)$ yielding thus the net profit condition.

The Sparre Andersen risk model introduced above has been investigated by numerous authors, see e.g., Gerber and Shiu (2005) and references therein. In practice, the surplus is also influenced by some additional uncertainties such as premium adjustments, legislation changes, cost of repairs, and other related expanses. Since various influences on the loss amounts cannot be modeled, a possible adequate modification is to add to the claim process another process which approximates all the unmodeled components of the aforementioned perturbations.

*Department of Actuarial Science, Faculty of Business and Economics, University of Lausanne, UNIL-Dorigny 1015 Lausanne, Switzerland 
Therefore, if $\{Y(t), t \geq 0\}$ is a real-valued stochastic process modeling the perturbation, the perturbed claim surplus process can be simply given as

$$
\widetilde{S}(t)=S(t)+Y(t), \quad t \geq 0 .
$$

Most of the models encountered in the literature assume that $Y$ is a standard Brownian motion (Bm) independent of $S$; in such cases $\widetilde{S}$ is a tractable jump-diffusion process. The motivation for choosing Bm as a perturbation comes from statistics; another motivation for choosing Bm is simply related to the fact that calculation of boundary crossing probabilities for Bm is straightforward when the boundary function is linear.

Since the introduction of the diffusion perturbed (Bm perturbed) risk model by Gerber (1970), the ruin probability of the perturbed risk model with various types of perturbations has become a popular theoretical topic in ruin theory. A seminal paper on the infinite-time ruin probability of the diffusion perturbed risk model is Dufresne and Gerber (1991), see also Yuen et al. (2005) and references therein.

Of course, instead of the Bm, general processes, including Lévy and Gaussian processes, can be considered as candidates for the perturbation, see e.g., Schlegel (1998), Furrer (1998) and Frostig (2008). For choosing a perturbation numerous processes can be considered, for instance one possible candidate could be an OrnsteinUhlenbeck process, another one could be a fractional Brownian motion (fBm) $\left\{B_{\alpha}(t), t \geq 0\right\}$, with parameter $\alpha \in(0,2]$, for which increments are positively correlated if $\alpha>1$, and negatively correlated if $\alpha<1$. However, the replacement of the $\mathrm{Bm}$ by a more general process $Y$ complicates the calculation of the ruin probability drastically, rendering the explicit formulas impossible.

A major drawback of the classical risk model is that claims are assumed to be settled immediately after their arrival. In practice this is clearly not the case: on one side claims are often reported with delay, such type of claims is commonly referred to as IBNR or simply incurred but not reported claims. Even when claims are reported, the case reserves might be over optimistic. Such claims are called incurred but not enough reserved (for short IBNER). The loss amount related to both IBNR and IBNER cannot be negligible, especially for TPL (third party liability) type covers. Accounting for those claims, a one-sided perturbation can be introduced as an approximation for the missing (unknown) part of the total claim amount, i.e., the total amount of the IBNR and IBNER claims. Furthermore, the insurers also do not know when IBNR and IBNER are ultimately settled. Therefore, it is reasonable to consider a time-changed process or an inflated process as perturbation. For instance, we can assume

$$
Y(t):=\left|B_{1}\left(R^{2} t\right)\right| \stackrel{d}{=} R\left|B_{1}(t)\right|, \quad t \geq 0,
$$

where $\left\{B_{1}\left(R^{2} t\right), t \geq 0\right\}$ is a time-changed standard $\mathrm{Bm}$, with some positive random variable $R$ (here $\stackrel{d}{=}$ stands for equality of distribution functions). Note further, in the light of the last expression, the random variable $R$ 
can also be interpreted as a random inflator introduced due to the delay in the settlement of the IBNR and IBNER claims.

Moreover, as previously mentioned, the additional perturbation process relates to different types of uncertainties such as premium adjustments, legislation changes, cost of repairs, IBNR or IBNER, and probably they are dependent, e.g., if there are more IBNR claims there will be more IBNER claims, and more expenses of dealing with them will be needed. With motivation from Constantinescu et al. (2011), where the authors considered a risk model with an Archimedean dependence structure modeling the dependence between claims, we model $d \in \mathbb{N}$ types of dependent uncertainties by a random vector process

$$
\vec{X}(t):=R\left(\left|W_{1}(t)\right|, \cdots,\left|W_{d}(t)\right|\right), \quad t \geq 0
$$

where $W_{1}, \ldots, W_{d}$ are independent copies of a centered stationary Gaussian process $\{W(t), t \geq 0\}$, with a.s. continuous sample paths and covariance function $r(t), t \geq 0$, and $R$ is a non-negative random variable modeling the dependence between different uncertainties. The model where $\{W(t), t \geq 0\}$ is a non-stationary Gaussian process is much more involved and will be considered in a forthcoming paper.

Based on the discussions above, we shall assume in the sequel that all the uncertainties are captured by a non-negative real-valued stochastic process given by

$$
Z_{+}(t):=R \chi_{d}(t)=R \sqrt{W_{1}^{2}(t)+\cdots+W_{d}^{2}(t)}, \quad t \geq 0
$$

which is independent of the claim surplus process $\{S(t), t \geq 0\}$. Moreover, we assume the random inflator $R$ is independent of $\chi_{d}$. It is worth noting that $\left\{Z_{+}(t), t \geq 0\right\}$ is a generalization of the inflated absolute value process (1.2), which gives us more degrees of freedom for modeling perturbations; also note that the perturbation by $Z_{+}^{2}$ can be studied with similar tools as those presented below for $Z_{+}$.

In this paper we consider the risk process perturbed by an inflated chi-process given by

$$
\tilde{S}(t)=S(t)+Z_{+}(t), \quad t \geq 0
$$

It turns out that the fluctuation caused by $Z_{+}$can be small or large depending on the distribution of $R$. Specifically, for any positive constant $T$, define finite-time ruin probabilities for the models (1.1) and (1.3) by

$$
\psi(u, T)=\mathbb{P}\left(\sup _{t \in[0, T]} S(t)>u\right) \quad \text { and } \tilde{\psi}(u, T)=\mathbb{P}\left(\sup _{t \in[0, T]} \tilde{S}(t)>u\right)
$$

respectively, where $u \geq 0$ is the initial reserve. Our main finding in this paper is that for certain models of the inflation and claim sizes

$$
\lim _{u \rightarrow \infty} \frac{\tilde{\psi}(u, T)}{\psi(u, T)}=1
$$


Further, we show that it is possible to specify both the distribution of the inflator and the distribution of claim sizes such that the ruin probability explained by the perturbed model is much higher compared to that derived by the classical (unperturbed) model, i.e.,

$$
\psi(u, T)=o(\tilde{\psi}(u, T)), \quad \text { as } u \rightarrow \infty
$$

The exact asymptotic behaviour of $\tilde{\psi}(u, T)$ is then derived in Theorem 2.1 below.

A nice review of early contributions on the finite-time ruin probability for the models without perturbation can be found in Tang (2004). In general, calculation of the finite-time ruin probability is more difficult than the infinite-time ruin probability. Therefore, often the aim of the analysis is to find adequate approximations for it by relying on the asymptotic theory.

In next section, we shall present the main result of this paper and then give two examples illustrating the applicability of our novel results. The proof of the main result together with some auxiliary lemmas will be relegated to Section 3.

\section{Main Result}

In this paper, we shall consider the claim sizes from the class $\mathcal{S}$ of subexponential distributions. By definition, a distribution function $F$ supported on $[0, \infty)$ is said to be in $\mathcal{S}$ if

$$
\lim _{x \rightarrow \infty} \frac{1-F^{* 2}(x)}{1-F(x)}=2,
$$

where $F^{* 2}$ denotes the 2 -fold convolution of $F$. It is known that the class $\mathcal{S}$ is in the class of heavy-tailed distributions. Furthermore, if $F \in \mathcal{S}$, then

$$
\lim _{x \rightarrow \infty} \frac{1-F(x)}{e^{-\epsilon x}}=\infty
$$

for any $\epsilon>0$; see Embrechts et al. (1997), Tang (2006), Li et al. (2010) and Foss et al. (2009,2011) for basic properties of subexponential distributions.

For the classical risk model, i.e., $\{N(t), t \geq 0\}$ is a homogeneous Poisson process with intensity parameter $\lambda \in(0, \infty)$, under the assumption $F \in \mathcal{S}$, it is known that

$$
\psi(u, T) \sim \lambda T(1-F(u))
$$

Here and in the following the symbol $\sim$ means that the quotient of both sides tends to 1 as the argument tends to infinity. Under some additional asymptotic assumptions on the distribution functions of the heavy-tailed claim sizes and of the inter-arrival times for the Sparre Andersen risk model defined in (1.1), Tang (2004) and 
Leipus and Šiaulys (2009) obtained novel asymptotic results for the finite-time ruin probabilities which hold uniformly for $T$ in some time interval. In addition, the finite-time ruin probabilities for some other non-standard risk models were considered in Gao and Wang (2010) and Yang et al. (2012) and references therein.

Our key assumption on the random inflator $R$ is that it has a Weibullian tail, i.e.,

$$
\mathbb{P}(R>x) \sim K x^{\beta} \exp \left(-L x^{p}\right), \quad x \rightarrow \infty
$$

with some constants $\beta \in \mathbb{R}$ and $K, L, p \in(0, \infty)$. Further, we impose two standard constrains on the correlation function $r(t)$ of the Gaussian process $\{W(t), t \geq 0\}$, namely,

(A1) $r(t)=1-a t^{\alpha}+o\left(|t|^{\alpha}\right)$ as $t \rightarrow 0$, with $\alpha \in(0,2]$ and some positive constant $a$, and

(A2) $r(t)<1$ for all $t>0$.

In the main result below we derive the exact asymptotics of the ruin probability for the perturbed risk model introduced above. It is surprising that, for $p \geq 2$ the claim of (1.4) can be confirmed, whereas the asymptotic behaviour of $\tilde{\psi}(u, T)$ is different (depending on the perturbation) for $p<2$, where additionally the well-known Pickands constant (Pickands (1969)) defined by

$$
\mathcal{H}_{\alpha}=\lim _{S \rightarrow \infty} S^{-1} \mathbb{E}\left(\exp \left(\sup _{t \in[0, S]}\left(\sqrt{2} B_{\alpha}(t)-t^{\alpha}\right)\right)\right), \quad \alpha \in(0,2]
$$

appears. Here $\left\{B_{\alpha}(t), t \geq 0\right\}$ is a $\mathrm{fBm}$ with Hurst parameter $\alpha / 2$. Pickands theorem, where Pickands constant first appears, plays a crucial role in the extremal behaviour of Gaussian processes. A rigorous proof of Pickands theorem is given in the seminal paper Piterbarg (1972), see also Piterbarg (1996).

For $u \geq 0$ set

$$
G(u):=\mathbb{P}\left(R \sup _{t \in[0, T]} \chi_{d}(t) \leq u\right)
$$

and recall that in our framework the random inflator $R$ is independent of the chi-process $\left\{\chi_{d}(t), t \geq 0\right\}$. Next, we present the main result of this contribution.

Theorem 2.1. For the perturbed Sparre Andersen risk model (1.3) with assumptions (2.7), (A1) and (A2), if there are two non-negative constants $\mu, \nu$ such that $\mu+\nu>0$, and further for some distribution function $H \in \mathcal{S}$ both $\psi(u, T) \sim \mu(1-H(u))$ and $1-G(u) \sim \nu(1-H(u))$ hold, then

$$
\max (\mu, \nu) \leq \liminf _{u \rightarrow \infty} \frac{\tilde{\psi}(u, T)}{1-H(u)} \leq \limsup _{u \rightarrow \infty} \frac{\tilde{\psi}(u, T)}{1-H(u)} \leq \mu+\nu .
$$

Furthermore,

(i) if $p \geq 2$ and $\mu>0$, then (1.4) holds;

(ii) if $0<p<2$ and $\mu=0$, then

$$
\tilde{\psi}(u, T) \sim\left(\frac{2 \pi}{p+2}\right)^{1 / 2} K T a^{1 / \alpha} \frac{2^{(2-d) / 2} \mathcal{H}_{\alpha}}{\Gamma(d / 2)}(p L)^{\frac{2+\alpha(d-1-\beta)}{\alpha(p+2)}} u^{\frac{2 \beta+p\left(\frac{2}{\alpha}+d-1\right)}{p+2}}
$$




$$
\times \exp \left(-\left(L^{\frac{2}{p+2}} p^{-\frac{p}{p+2}}+\frac{1}{2}(p L)^{\frac{2}{p+2}}\right) u^{\frac{2 p}{p+2}}\right),
$$

where $\Gamma(\cdot)$ is the Euler Gamma function.

Next, we present two illustrative examples.

Example 2.2. Assume that the claim inter-arrival times $\tau_{i}, i \in \mathbb{N}$, are exponentially distributed with parameter $\lambda>0$, i.e., $\mathbb{P}\left(\tau_{1}>x\right)=e^{-\lambda x}, x \geq 0$. Suppose further that the claim sizes $X_{i}, i \in \mathbb{N}$, are Weibull with distribution function $F(y)=1-\exp \left(-y^{\kappa}\right), y \geq 0$, and shape parameter $\kappa \in(0,1), R \stackrel{d}{=}|\mathcal{N}|$ with $\mathcal{N}$ being the standard normal random variable, and $W(t)=B(t+1)-B(t)$ with $\{B(t), t \geq 0\}$ a standard Bm. It follows that $\{W(t), t \geq 0\}$ is a stationary Gaussian process (also referred to as Slepian process) with correlation function $r(t)$ satisfying

$$
r(t)=1-t+o(t), \quad \text { as } \quad t \rightarrow 0
$$

and $r(t)<1$ for any $t>0$. Moreover, we have $p=2$ since

$$
\mathbb{P}(R>u)=(1+o(1)) \sqrt{\frac{2}{\pi}} u^{-1} e^{-\frac{1}{2} u^{2}} .
$$

Consequently, in view of (2.6) and Theorem 2.1 (i) we conclude that

$$
\tilde{\psi}(u, T) \sim \psi(u, T) \sim \lambda T e^{-u^{\kappa}}, \quad \text { as } \quad u \rightarrow \infty .
$$

Example 2.3. Assume that the claim sizes $X_{i}, i \in \mathbb{N}$, are exponentially distributed with parameter $\delta>0, R$ is exponentially distributed with parameter $L>0$, and $\{W(t), t \geq 0\}$ is an Ornstein-Uhlenbeck process with correlation function

$$
r(t)=e^{-t}, \quad t \geq 0 .
$$

It is known (e.g., Rolski et al. (1999) p.251) that the infinite-time ruin probability for the Sparre Andersen risk model with exponential claims is given by

$$
\psi(u, \infty)=\frac{\delta-s_{+}}{\delta} e^{-s_{+} u}, \quad u \geq 0
$$

where $s_{+}$is the unique positive root of

$$
\frac{\delta}{\delta-s} \hat{L}_{\tau}(c s)=1
$$

with $\hat{L}_{\tau}(s)=\int_{0}^{\infty} e^{-s x} d F_{\tau}(x)$ the Laplace transform of the distribution $F_{\tau}$. Therefore, by the fact that

$$
\psi(u, T)=\mathbb{P}\left(\sup _{t \in[0, T]} S(t)>u\right) \leq \mathbb{P}\left(\sup _{t \in[0, \infty)} S(t)>u\right)=\psi(u, \infty)
$$


for any $u>0$, we conclude that

$$
\psi(u, T) \leq \frac{\delta-s_{+}}{\delta} e^{-s_{+} u}, \quad u \geq 0
$$

which means that $\mu=0$. Furthermore, we have

$$
r(t)=1-t+o(t), \quad \text { as } \quad t \rightarrow 0
$$

and $r(t)<1$ for any $t>0$. In addition, $p=1$ follows from

$$
\mathbb{P}(R>u)=e^{-L u} .
$$

Consequently, Theorem 2.1 (ii) implies (note that $\mathcal{H}_{1}=1$ )

$$
\tilde{\psi}(u, T) \quad \sim \quad\left(\frac{2 \pi}{3}\right)^{1 / 2} \frac{2^{(2-d) / 2}}{\Gamma(d / 2)} T(L u)^{\frac{d+1}{3}} e^{-\frac{3}{2}(L u)^{\frac{2}{3}}}, \quad \text { as } \quad u \rightarrow \infty .
$$

\section{Further Results and Proofs}

We first present a crucial theorem on the supremum of chi-process given in Corollary 7.3 in Piterbarg (1996).

Theorem 3.1. Suppose that the covariance function $r(t)$ of the centered Gaussian process $\{W(t), t \geq 0\}$ satisfies assumptions (A1) and (A2) with $\alpha \in(0,2]$ and some positive constant a. If $\chi_{d}(t)=\sqrt{W_{1}^{2}(t)+\cdots+W_{d}^{2}(t)}$ is the chi-process generated from the i.i.d. copies $W_{1}, \ldots, W_{d}$ of $W$, then

$$
\mathbb{P}\left(\sup _{t \in[0, T]} \chi_{d}(t)>u\right) \sim T a^{1 / \alpha} \frac{2^{(2-d) / 2} \mathcal{H}_{\alpha}}{\Gamma(d / 2)} u^{2 / \alpha+d-2} \exp \left(-\frac{u^{2}}{2}\right)
$$

holds as $u \rightarrow \infty$, with $\mathcal{H}_{\alpha}$ defined in (2.8).

The following result is given in Lemma 2.1 of Arendarczyk and Dȩbicki (2011).

Lemma 3.2. Let $R_{i}, i=1,2$ be two independent non-negative random variables. If for $x \rightarrow \infty$,

$$
\mathbb{P}\left(R_{i}>x\right) \sim C_{i} x^{\alpha_{i}} \exp \left(-L_{i} x^{p_{i}}\right), \quad i=1,2,
$$

with some constants $\alpha_{i} \in \mathbb{R}$ and $C_{i}, L_{i}, p_{i} \in(0, \infty), i=1,2$, then we have

$$
\begin{aligned}
\mathbb{P}\left(R_{1} R_{2}>x\right) \sim & \left(\frac{2 \pi p_{2} L_{2}}{p_{1}+p_{2}}\right)^{1 / 2} C_{1} C_{2} A^{p_{2} / 2+\alpha_{2}-\alpha_{1}} x^{\frac{2 p_{2} \alpha_{1}+2 p_{1} \alpha_{2}+p_{1} p_{2}}{2\left(p_{1}+p_{2}\right)}} \\
& \times \exp \left(-\left(L_{1} A^{-p_{1}}+L_{2} A^{p_{2}}\right) x^{\frac{p_{1} p_{2}}{p_{1}+p_{2}}}\right),
\end{aligned}
$$

where $\left.A=\left(p_{1} L_{1}\right) /\left(p_{2} L_{2}\right)\right]^{1 /\left(p_{1}+p_{2}\right)}$. 
Lemma 3.3. Suppose that the random inflator $R>0$ satisfies (2.7) with some constants $\beta \in \mathbb{R}$ and $K, L, p \in$ $(0, \infty)$. If additionally $R$ is independent of the chi-square process $\chi_{d}$, then under the assumptions of Theorem 3.1, we obtain

$$
\begin{aligned}
1-G(u)=\mathbb{P}\left(R \sup _{t \in[0, T]} \chi_{d}(t)>u\right) \sim & \left(\frac{2 \pi}{p+2}\right)^{1 / 2} K T a^{1 / \alpha} \frac{2^{(2-d) / 2} \mathcal{H}_{\alpha}}{\Gamma(d / 2)}(p L)^{\frac{2+\alpha(d-1-\beta)}{\alpha(p+2)}} u^{\frac{2 \beta+p(2 / \alpha+d-1)}{p+2}} \\
& \times \exp \left(-\left(L^{\frac{2}{p+2}} p^{-\frac{p}{p+2}}+\frac{1}{2}(p L)^{\frac{2}{p+2}}\right) u^{\frac{2 p}{p+2}}\right)
\end{aligned}
$$

Furthermore, if $0<p<2$, then $G$ is a subexponential distribution in the Gumbel max-domain of attraction.

Proof of Lemma 3.3 The exact asymptotics in (3.14) follows from Theorem 3.1 and Lemma 3.2. It is clear that $G$ is in the Gumbel max-domain of attraction, and for $0<p<2$ it is also a subexponential distribution.

Proof of Theorem 2.1 For any $u \geq 0$

$$
\begin{aligned}
\tilde{\psi}(u, T) & \leq \mathbb{P}\left(\sup _{t \in[0, T]} S(t)+\sup _{t \in[0, T]} R \chi_{d}(t)>u\right) \\
& =1-(1-\psi(\cdot, T)) * G(u) \\
& \sim(\mu+\nu)(1-H(u)),
\end{aligned}
$$

where $(1-\psi(\cdot, T)) * G(u)$ is the convolution function of the functions $1-\psi(u, T)$ and $G(u)$. Hence we conclude that

$$
\limsup _{u \rightarrow \infty} \frac{\tilde{\psi}(u, T)}{1-H(u)} \leq \mu+\nu
$$

establishing thus the last inequality of (2.9). For the first inequality of (2.9), without loss of generality, we prove the result for $\mu>0$. For any $u \geq 0$ we have

$$
\begin{aligned}
\tilde{\psi}(u, T) & \geq \mathbb{P}\left(\sup _{t \in[0, T]} S(t)-\sup _{t \in[0, T]}\left(-R \chi_{d}(t)\right)>u\right) \\
& \geq \mathbb{P}\left(\sup _{t \in[0, T]} S(t)>u\right) \\
& \sim \psi(u, T) \\
& \sim \mu(1-H(u))
\end{aligned}
$$

and thus

$$
\mu \leq \liminf _{u \rightarrow \infty} \frac{\tilde{\psi}(u, T)}{1-H(u)}
$$

In view of $(2.5)$ and Lemma 3.3 we see that $\nu=0$ when $p \geq 2$. In fact, if we suppose that $\nu>0$, then

$$
\lim _{u \rightarrow \infty} \frac{1-G(u)}{e^{-\epsilon u}} \frac{e^{-\epsilon u}}{1-H(u)}=\lim _{u \rightarrow \infty} \frac{1-G(u)}{1-H(u)}=\nu>0
$$


for any sufficiently small $\epsilon>0$, which by (2.5) does not hold when $p \geq 2$. Consequently, (1.4) follows immediately from (2.9). The claim in (2.10) follows from (2.9) and Lemma 3.3, and thus the proof is completed.

Acknowledgments. We would like to thank the referee for numerous comments and suggestions which significantly improved this contribution. We would also like to thank Professor Krzysztof Dębicki and Professor Qihe Tang for several discussions and suggestions. Both authors kindly acknowledge partial support by the Swiss National Science Foundation Grant 200021-1401633/1.

\section{References}

[1] Arendarczyk, M., Dębicki, K., 2011. Asymptotics of supremum distribution of a Gaussian process over a Weibullian time. Bernoulli 17, 194-210.

[2] Constantinescu, C., Hashorva, E., Ji, L., 2011. Archimedean copulas in finite and infinite dimensions - with application to ruin problems. Insurance: Mathematics and Economics 49(3), 487-495.

[3] Dufresne, F., Gerber, H.U., 1991. Risk theory for the compound Poisson process that is perturbed by diffusion. Insurance: Mathematics and Economics 10, 51-59.

[4] Embrechts, P., Klüpelberg, C., Mikosch, T., 1997. Modeling extremal events for finance and insurance. Berlin, Springer.

[5] Frostig, E., 2008. On ruin probability for a risk process perturbed by a Lévy process with no negative jumps. Stochastic Models 24 (2), 288-313.

[6] Foss, S., Korshunov, D., Zachary, S., 2009. Convolutions of long-tailed and subexponential distributions. J. Appl. Probab. 46, 756-767.

[7] Foss, S., Korshunov, D., Zachary, S., 2011. An Introduction to Heavy-tailed and Subexponential Distributions. Springer-Verlag, New York.

[8] Furrer, H.J., 1998. Risk processes perturbed by a-stable Lévy motion. Scandinavian Actuarial Journal 10, 23-35.

[9] Gao, Q., Wang, Y., 2010. Randomly weighted sums with dominated varying-tailed increments and application to risk theory. Journal of the Korean Statistical Society 39, 305-314.

[10] Gerber, H.U., Shiu, E.S.W., 2005. The time value of ruin in a Sparre Andersen model. North American Actuarial Journal 9, 49-69.

[11] Gerber, H.U., 1970. An extension of the renewal equation and its application in the collective theory of risk. Skandinnavisk Aktuarietidskrift 205-210. 
[12] Leipus, R., Šiaulys, J., 2009. Asymptotic behaviour of the finite-time ruin probability in renewal risk models. Appl. Stochastic Models Bus. Ind. 25, 309-321.

[13] Li, J., Tang, Q., Wu, R., 2010. Subexponential tails of discounted aggregate claims in a time-dependent renewal risk model. Adv. Appl. Probab. 42, 1126-1146.

[14] Liu, Y., and Tang, Q., 2010. Subexponentiality of the product convolution of two Weibull-type distributions. J. Aust. Math. Soc. 89, 277-288.

[15] Pickands, J. III., 1969. Asymptotic properties of the maximum in a stationary Gaussian process. Transactions of the American Mathematical Society 145, 75-86.

[16] Piterbarg, V., 1972. On the paper by J. Pickands "Upcrosssing probabilities for stationary Gaussian processes", Vestnik Moscow. Univ. Ser. I Mat. Mekh. 27, 25-30. English transl. in Moscow Univ. Math. Bull., 27 (1972).

[17] Piterbarg, V., 1996. Asymptotic Methods in the Theory of Gaussian Processes and Fields. In: Transl. Math. Monographs, vol. 148. AMS, Providence, RI.

[18] Rolski, T., Schmidli, H., Schmidt, V., and Teugels, J.L., 1999. Stochastic processes for insurance and finance. John Wiley and Sons, Chichester.

[19] Schlegel, S., 1998. Ruin probabilities in perturbed risk models. Insurance: Mathematics and Economics 22, 93-104.

[20] Tang, Q., 2004. Asymptotics for the finite time ruin probability in the renewal model with consistent variation. Stochastic Models 20, 281-297.

[21] Tang, Q., 2006. On convolution equivalence with applications. Bernoulli, 12, 535-549.

[22] Yang, Y., Lin. J., Huang, C. and Ma, X., 2012. The finite-time ruin probability in two non-standard renewal risk models with constant interest rate and dependent subexponential claims. Journal of the Korean Statistical Society 41, 213-224.

[23] Yuen, K., Yang, H. and Wang, R., 2005. On Erlang(2) risk process perturbed by diffusion. Communications in Statistics-Theory and Methods 34, 2197-2208. 